\title{
Diatomáceas de rios da bacia hidrográfica Litorânea, PR, Brasil: Coscinodiscophyceae e Fragilariophyceae
}

\author{
Monaliza Landucci ${ }^{1}$ e Thelma A. Veiga Ludwig ${ }^{1,2}$
}

Recebido em 03/07/2003. Aceito em 08/10/2004

\begin{abstract}
RESUMO - (Diatomáceas de rios da bacia hidrográfica Litorânea, PR, Brasil: Coscinodiscophyceae e Fragilariophyceae). Realizou-se o inventário florístico das diatomáceas pertencentes às classes Coscinodiscophyceae e Fragilariophyceae de rios da bacia hidrográfica Litorânea do Paraná. Amostras planctônicas e perifíticas foram coletadas em dezembro/1998, e em fevereiro e julho/2000 em nove rios: Iporanga, Nhundiaquara, do Nunes, Cachoeira, do Salto, Cambará, da Onça, Cabaraquara e do Engenho. Identificaram-se 20 táxons infragenéricos, sendo Aulacoseira distans (Ehr.) Sim. var. distans citação pioneira para o Estado do Paraná. Foram de ocorrência exclusiva em amostras planctônicas Asterionella formosa Hass. var. formosa e Cyclotella stelligera (Cl. \& Grun.) V. Heurck var. stelligera. Por outro lado, Cyclotella pseudostelligera Hust. var. pseudostelligera e Fragilaria capucina var. vaucheriae (Kütz.) Lang.-Bert. foram de ocorrência exclusiva em amostras perifíticas.
\end{abstract}

Palavras-chave: Diatomáceas, taxonomia, fitoplâncton, perifíton, ambiente lótico

ABSTRACT - (Diatoms from Litorânea watershed rivers, Paraná State, Brazil: Coscinodiscophyceae and Fragilariophyceae). The survey is a floristic inventory of Coscinodiscophyceae and Fragilariophyceae diatoms from lotic systems of Litorânea watershed, east Paraná, Brazil. Diatoms were collected from periphytic and planktonic microhabitats on December 1998, February and July 2000. Nine rivers were sampled: Iporanga, Nhundiaquara, Nunes, Cachoeira, Salto, Cambará, Onça, Cabaraquara and Engenho. Twenty taxa were identified. Aulacoseira distans (Ehr.) Sim. var. distans was first recorded in the State. Asterionella formosa Hass. var. formosa and Cyclotella stelligera (Cl. \& Grun.) V. Heurck var. stelligera occurred only at planktonic samples and Cyclotella pseudostelligera Hust. var. pseudostelligera e Fragilaria capucina var. vaucheriae (Kütz.) Lang.-Bert. were exclusively registered at periphytic ones.

Key words: Diatoms, taxonomy, phytoplancton, periphyton, lotic system

\section{Introdução}

A bacia hidrográfica Litorânea, localizada a leste do Estado do Paraná, abrange área de drenagem de $5.766 \mathrm{~km}^{2}$. Compreende a serra do Mar, a planície Costeira e as baías de Paranaguá e Guaratuba (Bigarella 1978). Mesmo constituindo-se em uma vasta bacia hidrográfica, não há registros de trabalhos sobre a diatomoflórula de ambientes lóticos dulcícolas nesta bacia.

O estudo da diatomoflórula de ambientes lóticos continentais paranaenses iniciou-se na bacia do rio Iguaçu: Contin (1990) realizou amostragem na região da barragem de captação d’água deste rio; Ludwig \& Flôres (1995; 1997), em rios a serem represados para a construção da usina hidrelétrica de Segredo e Brassac et al. (1999) identificaram as diatomáceas cêntricas de rios da região de abrangência da usina hidrelétrica de Salto Caxias. Bittencourt-Oliveira (2002), em trabalho sobre a comunidade fitoplanctônica do rio Tibagi, registrou 52 diatomáceas. Espécies perifíticas de Navicula foram estudadas por Leandrini et al. (2002), em amostras do rio Pirapó e córrego Sarandi, na região de Maringá.

Parte de um amplo projeto que visa o inventário florístico de ambientes lóticos do Estado do Paraná, este subprojeto objetiva contribuir com a taxonomia e distribuição geográfica das diatomáceas pertencentes às classes Coscinodiscophyceae e Fragilariophyceae de rios da bacia Litorânea paranaense.

\section{Material e métodos}

O litoral do Estado do Paraná estende-se desde a vila do Ararapira ao norte (2512'44”' S - 4801'15'” W Gr) até a barra do Saí-Guaçu, ao sul (255' $38^{\circ}$ ' S -

\footnotetext{
1 Universidade Federal do Paraná, UFPR, Departamento de Botânica, Setor de Ciências Biológicas, C. Postal 19031, CEP 81531-990, Curitiba, PR, Brasil

2 Autor para correspondência: veiga@ufpr.br
} 
48³5’26” W Gr). A bacia hidrográfica Litorânea localiza-se a leste do Estado, com rios percorrendo a área serrana e desembocando na planície costeira. Nove rios foram selecionados de maneira a abranger os diferentes municípios da região: Iporanga, Nhundiaquara, do Nunes, Cachoeira, do Salto, Cambará, da Onça, Cabaraquara e do Engenho.

Amostras perifíticas e planctônicas foram coletadas em cada rio, totalizando 18 amostras para estudo. As amostras fitoplanctônicas foram obtidas por meio de rede de plâncton com $25 \mu \mathrm{m}$ de abertura de malha, permitindo-se a passagem de água corrente por aproximadamente 10 minutos, sendo fixadas com solução de formol na proporção de $4 \% \mathrm{v} / \mathrm{v}$.

Substratos disponíveis tais como partes submersas de macrófitas aquáticas, folhas e seixos soltos no leito dos rios, foram colocados diretamente em frascos contendo solução de formol (1 parte para 25 partes de água), constituindo as amostras perifíticas. Em laboratório, procedeu-se à raspagem do material aderido, com auxílio de escovas com cerdas macias e lâminas cortantes, para posterior montagem de lâminas e oxidação da matéria orgânica.

O estudo qualitativo baseou-se em duas séries de lâminas permanentes, uma com material fresco e outra com amostras submetidas à oxidação (Simonsen 1974, modificada por Moreira Filho \& Valente-Moreira 1981).
O enquadramento taxonômico seguiu Round et al. (1991).

As amostras líquidas, assim como as respectivas lâminas permanentes, encontram-se tombadas no Herbário da Universidade Federal do Paraná (UPCB).

\section{Resultados e discussão}

O estudo florístico das diatomáceas Coscinodiscophyceae e Fragilariophyceae da bacia hidrográfica Litorânea permitiu a identificação de 20 táxons infragenéricos, citando pela primeira vez Aulacoseira distans var. distans para o Estado do Paraná. Foram de ocorrência exclusiva em amostras planctônicas Asterionella formosa var. formosa e Cyclotella stelligera var. stelligera. Por outro lado, Cyclotella pseudostelligera var. pseudostelligera e Fragilaria capucina var. vaucheriae foram de ocorrência exclusiva em amostras perifíticas. Dentre os táxons inventariados, $70 \%$ foram registrados tanto em amostras planctônicas quanto nas perifíticas.

Os 20 táxons infragenéricos identificados estão distribuídos em nove gêneros e seis famílias, como segue:

Classe Coscinodiscophyceae Round \& Crawford

Ordem Thalassiosirales Glezer \& Makorova

Família Stephanodiscaceae Glezer \& Makorova

Cyclotella (Kützing) Brëbisson

Chave para os táxons de Cyclotella

1. Área central hialina ou pontuada, sem aréola central isolada ........ 1. C. meneghiniana var. meneghiniana

1. Área central ornamentada, presença de aréola central isolada circundada por estrias radiadas

2. Presença de processos marginais, diâmetro inferior a $6 \mu \mathrm{m}$...... 2. C. pseudostelligera var. pseudostelligera

2. Ausência de processos marginais, diâmetro superior a $10 \mu \mathrm{m}$ 3. C. stelligera var. stelligera

1. Cyclotella meneghiniana Kützing var. meneghiniana, Bacill., p. 50, pl. 30: 68. 1844.

Fig. 3-5.

Valvas circulares, área central hialina ou delicadamente pontuada, podendo ou não apresentar uma ou mais pórtulas; região marginal com estrias alveoladas, robustas, radiadas. Diâmetro: 6,4-16,2 $\mu \mathrm{m}$; estrias: 8 a 12 em $10 \mu \mathrm{m}$.

Registrada a ocorrência de espécimes em amostras planctônicas e perifíticas.

Material examinado: BRASIL. Paraná: Morretes, rio Iporanga, perifíton, loc. de América de Cima, próximo à estação de tratamento de água da SANEPAR,
18/III/2000, M. Landucci \& E.L. Corrêa (UPCB44533); Antonina, rio do Nunes, perifíton, rodovia PR-340, 25/III/2000, M. Landucci \& E.L. Corrêa (UPCB44537); Paranaguá, rio Cambará, perifíton, rodovia Alexandra-Matinhos (PR-508), Colônia Cambará, 1/IV/2000, M. Landucci \& E.L. Corrêa (UPCB44543); fitoplâncton, rodovia AlexandraMatinhos (PR-508), Colônia Cambará, 1/IV/2000, $M$. Landucci \& E.L. Corrêa (UPCB44544).

2.Cyclotella pseudostelligera Hustedt var. pseudostelligera, Abh. Nat. Ver. Bremen.: 581, f. 1-2. 1939.

Fig. 8-10. 
Valvas circulares, área central com aréola isolada, circundada por estrias radiadas, encurtadas irregularmente formando uma roseta; região marginal com estrias alveoladas radiadas, regularmente encurtadas e processos marginais (fultopórtulas) conspícuos. Diâmetro: 4,1-5 $\mu \mathrm{m}$; estrias: 14-18 em $10 \mu \mathrm{m}$, processos marginais: 5-6 em $10 \mu \mathrm{m}$.

Os espécimes de $C$. pseudostelligera, registrados em amostra perifítica para a região litorânea do Estado, apresentaram anel de processos marginais grosseiros e tamanho diminuto das valvas, concordando com a circunscrição apresentada por Huber-Pestalozzi (1942).

Material examinado: BRASIL. Paraná: munic. Morretes, rio Iporanga, perifíton, loc. de América de Cima, próximo à estação de tratamento de água da SANEPAR, 18/III/2000, M. Landucci \& E.L. Corrêa (UPCB44533); Antonina, rio do Nunes, perifíton, rodovia PR-340, 25/III/2000, M. Landucci \& E.L. Corrêa (UPCB44537); Paranaguá, rio Cambará, perifíton, rodovia Alexandra-Matinhos (PR-508),
Colônia Cambará, 1/IV/2000, M. Landucci \& E.L. Corrêa (UPCB44543).

3. Cyclotella stelligera (Cleve \& Grunow) Van Heurck var. stelligera, Syn. Diat. Beig., pl. 94, fig. 22-26. 1884.

Fig. 7.

Valvas circulares, área central com aréola isolada circundada por estrias encurtadas irregularmente, formando uma roseta; região marginal com estrias alveoladas regularmente encurtadas, radiadas. Diâmetro: 11,5-18,6 $\mu \mathrm{m}$; estrias: 7-13 em $10 \mu \mathrm{m}$.

A população estudada foi registrada em uma única amostra, de origem planctônica.

Material examinado: BRASIL. Paraná: Paranaguá, rio do Salto, fitoplâncton, loc. de Quintilha, Rodovia PR-508, 1/IV/2000, M. Landucci \& E.L. Corrêa (UPCB44542).

Ordem Orthoseirales Crawford

Família Orthoseiraceae Crawford

Orthoseira Thwaites

\section{Chave para os táxons de Orthoseira}

1. Vista pleural com bandas retangulares; estrias areoladas, em fileiras retas a levemente oblíquas

1. O. dendroteres var. dendroteres

1. Vista pleural com bandas espirais; estrias delicadamente areoladas, em fileiras oblíquas

2. O. roeseana var. roeseana

1. Orthoseira dendroteres (Ehrenberg) Crawford var. dendroteres, Phycologia, 20(2): 174-192. 1991.

Fig. 6, 13.

Valvas circulares, superfície plana apresentando ondulações na margem, área central com carinopórtulas; estrias areoladas formando fileiras radiadas, regulares. Vista pleural formando bandas espirais, apresentando estrias delicadamente areoladas, dispostas obliquamente. Diâmetro: 12,1-13,7 $\mu$ m; estrias: 13-15 em $10 \mu \mathrm{m}$; aréolas: 20-24 em 10 m; carinopórtulas: 3 em $10 \mu \mathrm{m}$.

Krammer \& Lange-Bertalot (1991) comentaram que O. roeseana (Rabenhrost) O’Meara é semelhante a $O$. dendroteres, diferindo-as pelos espinhos de ligação curtos, na primeira, e longos em $O$. dendroteres. Porém, os espinhos podem ser danificados no processo de oxidação e limpeza das frústulas, havendo necessidade de observar espécimes que não passaram pelo processo químico. Neste caso, outras características morfológicas podem não estar bem evidenciadas. Além disso, a visualização dos espinhos pode estar dificultada pelo fato das células serem muito reduzidas ou pela curvatura do manto valvar. Esse critério não tem se mostrado eficiente como caráter taxonômico diagnóstico.

Houk (1993) caracterizou o complexo Liparogyra dendroteres Ehrenberg pela presença de cíngulo relativamente longo, com mais de 13 bandas, geralmente onduladas, manto ornado por estrias densas e presença de espinhos nas margens da superfície valvar. Sobre $O$. roeseana, o autor mencionou que espinhos marginais também podem estar presentes e que o cíngulo possui de 3 a 5 bandas. Spaulding \& Kociolek (1998) afirmaram que o uso da morfologia dos espinhos, a plasticidade morfológica da frústula e a problemática nomenclatural existente no grupo, impossibilitam a identificação de seus espécimes em nível infra-genérico. As determinações dos espécimes 
da bacia Litorânea foram possíveis utilizando-se os critérios de Houk (1993), além da razão comprimento/ largura da frústula. Verificou-se uma semelhança entre os exemplares estudados e os indivíduos da população da usina hidrelétrica de Salto Caxias (Brassac et al. 1999). Entretanto, ressalta-se a importância de estudos que visem a elucidação da problemática taxonômica envolvendo o complexo "Orthoseira Thwaites”.

Material examinado: BRASIL. Paraná: Morretes, rio Nhundiaquara, fitoplâncton, Vila Freitas, rodovia PR-408, 25/III/2000, M. Landucci \& E.L. Corrêa (UPCB44536); Antonina, rio do Nunes, perifíton, rodovia PR-340, 25/III/2000, M. Landucci \& E.L. Corrêa (UPCB44537).

2. Orthoseira roeseana (Rabenhrost) O'Meara var. roeseana, Proc. Roy. Irich. Acad., 2: 255, 1876.

Fig. 1-2.

Valvas circulares, superfície plana apresentando espinhos marginais conspícuos; área central com carinopórtulas; estrias areoladas formando fileiras irregulares, radiadas. Vista pleural formando bandas retangulares, apresentando estrias areoladas, dispostas em fileira retas a levemente oblíquas. Diâmetro: 17,8-37,8 $\mu \mathrm{m}$; estrias: 10-26 em $10 \mu \mathrm{m}$; aréolas: 22-26 em $10 \mu \mathrm{m}$; carinopórtulas: 3 em $10 \mu \mathrm{m}$.

Foram encontrados exemplares em amostras planctônicas e perifíticas. Ver comentários em O. dendroteres.

Material examinado: BRASIL. Paraná: Morretes, rio Iporanga, perifíton, loc. de América de Cima, próximo à estação de tratamento de água da SANEPAR, 18/III/2000, M. Landucci \& E.L. Corrêa (UPCB44533); fitoplâncton, loc. de América de Cima, próximo à estação de tratamento de água da SANEPAR 18/III/2000, coletor: M. Landucci \& E.L. Corrêa (UPCB44534); rio Nhundiaquara, perifíton, Vila Freitas, rodovia PR-408, 25/III/2000, M. Landucci \& E.L. Corrêa (UPCB44535); Antonina, rio do Nunes, fitoplâncton, rodovia PR-340, 25/III/2000, M. Landucci \& E.L. Corrêa (UPCB44538); rio Cachoeira, perifíton, loc.de Bairro Alto, 25/III/2000, M. Landucci \& E.L. Corrêa (UPCB44539).

Ordem Melosirales Crawford

Família Melosiraceae Kützing

Melosira C.A. Agardh

Melosira varians Agardh var. varians, Bot. Zeit., p. 628, 1827.

Fig. 17-18.
Frústulas cilíndricas em vista pleural, formando cadeias filamentosas retas, unidas por coroa marginal de espinhos inconspícuos; sulco ausente, pseudo-sulco presente; manto ornamentado por aréolas delicadas, inconspícuas. Altura da célula: 16,2-27,5 $\mu \mathrm{m}$; diâmetro: 12,1-14,2 $\mu \mathrm{m}$.

Melosira varians Agardh ocorreu tanto em amostras planctônicas quanto perifíticas.

Material examinado: BRASIL. Paraná: Morretes, rio Nhundiaquara, perifíton, Vila Freitas, rodovia PR-408, 25/III/2000, M. Landucci \& E.L. Corrêa (UPCB44535); fitoplâncton, Vila Freitas, rodovia PR408, 25/III/2000, M. Landucci \& E.L. Corrêa (UPCB44536); Antonina, rio do Nunes, perifíton, rodovia PR-340, 25/III/2000, M. Landucci \& E.L. Corrêa (UPCB44537); fitoplâncton, rodovia PR-340, 25/III/2000, M. Landucci \& E.L. Corrêa (UPCB44538); rio Cachoeira, perifíton, loc.de Bairro Alto, 25/III/2000, M. Landucci \& E.L. Corrêa (UPCB44539); fitoplâncton, loc.de Bairro Alto, 25/III/2000, M. Landucci \& E.L. Corrêa (UPCB44540); Paranaguá, rio do Salto, perifíton, loc.de Quintilha, Rodovia PR-508, 1/IV/2000, M. Landucci \& E.L. Corrêa (UPCB44541); fitoplâncton, loc. de Quintilha, Rodovia PR-508, 1/IV/2000, M. Landucci \& E.L. Corrêa (UPCB44542).

Ordem Biddulphiales Krieger

Família Biddulphiaceae Kützing

Hydrosera G.C. Wallich

Hydrosera whampöensis (Schwartz) Deby var. whampöensis, J. Microgr., pr. 15, p. 232-240, 1891.

Fig. 24a.

Valvas triangulares apresentando margens onduladas; ângulos arredondados com distintos campos de poros apicais, separados internamente por pseudoseptos; superfície valvar ornamentada por aréolas irregulares. Diâmetro: 56,7×68-68,8×86,6 ㅆm; estrias: 5-9 em $10 \mu \mathrm{m}$.

Deby (1891) descreveu Hydrosera whampöensis, mencionando a presença de apêndices valvares largamente arredondados e mais largos do que longos. Comentou ainda que $H$. triquetra Wallich var. triquetra, espécie semelhante, caracteriza-se pelos apêndices cônicos e mais longos do que largos, cujos ângulos valvares são mais fortemente protraídos em direção ao exterior, conferindo-lhe uma forma mais triangular. Domitrovic \& Maidana (1997) apresentaram exemplares de $H$. whampoensis com contorno valvar triangular e pólos arredondados. Os autores relataram 
a distribuição geográfica da espécie para a Argentina, China e Tasmânia, destacando ainda a ocorrência desta para águas paranaenses, principalmente em ambientes reófilos, porém sem evidenciar exemplares com plastos. Os exemplares analisados apresentaram contorno valvar triangular e pólos arredondados, ocorreram em amostras planctônicas e perifíticas do rio do Salto localizado no município de Paranaguá e foi possível observar plastos no interior das frústulas.

Material examinado: BRASIL. Paraná: Parana- guá, rio do Salto, perifíton, loc.de Quintilha, Rodovia PR-508, 1/IV/2000, M. Landucci \& E.L. Corrêa (UPCB44541); fitoplâncton, loc. de Quintilha, Rodovia PR-508, 1/IV/2000, M. Landucci \& E.L. Corrêa (UPCB44542); Guaraqueçaba, rio do Engenho, fitoplâncton, reserva Salto Morato, 2/IV/2000, $M$. Landucci \& D. Atab (UPCB44550).

Ordem Aulacoseirales Crawford

Família Aulacoseiraceae Crawford

Aulacoseira Thwaithes

Chave para os táxons de Aulacoseira

1. Valvas unidas por um espinho longo, geralmente ocorrendo nas células terminais do filamento, e por vários espinhos curtos de ligação

2. Diâmetro valvar inferior a $3,5 \mu \mathrm{m}$

4. A. granulata var. angustissima

2. Diâmetro valvar superior a 5,5 $\mu \mathrm{m}$ 5. A. granulata var. granulata

1. Valvas unidas apenas por espinhos curtos de ligação

3. Eixo pervalvar superior a $19,0 \mu \mathrm{m}$ 2. A. ambigua var. ambigua f. ambigua

3. Eixo pervalvar inferior a $12,0 \mu \mathrm{m}$

4. Vista valvar com aréolas presente em toda superfície, vista pleural com estrias dispostas paralelamente em relação ao eixo valvar 3. A. distans var. distans

4. Vista valvar com aréolas presente apenas nas margens, vista pleural com estrias dispostas de maneira obliqua ou levemente oblíqua em relação ao eixo valvar 1. A. alpigena var. alpigena

1. Aulacoseira alpigena (Grunow) Krammer var. alpigena, In Pascher, Süssw, FI. Mitteleur., v.2, n.3, p. 34, fig. 34, fig. 2: 3-7: 1-25, 32: 10-16. 1991.

Fig. 11-12.

Frústulas cilíndricas em vista pleural, formando cadeias retas, curtas, unidas por espinhos de ligação curtos, inconspícuos; presença de sulco e pseudosulco pouco profundos; manto ornamentado por estrias areoladas delicadas, dispostas de maneira obliqua ou levemente oblíqua em relação ao eixo pervalvar. Em vista valvar as aréolas estão presentes apenas na margem da valva. Eixo pervalvar: 4,4-11,2 $\mu$ m; diâmetro: 4,0-5,6 بm; estrias: 20-24 em $10 \mu \mathrm{m}$.

O material determinado é coincidente com os apresentados por Hustedt (1927-1930), Cleve-Euler (1951) e Krammer \& Lange-Bertalot (1991). De acordo com Krammer \& Lange-Bertalot (1991), Aulacoseira alpigena apresenta estrias dispostas de maneira obliqua ou levemente oblíqua e vista valvar com aréolas apenas na margem da valva. Aulacoseira distans (Ehrenberg) Simonsen apresenta estrias dispostas paralelamente e vista valvar com aréolas bem definidas e distribuídas em toda sua superfície.
Representantes da espécie foram encontrados em amostras planctônicas e perifíticas.

Material examinado: BRASIL. Paraná: Paranaguá, rio Cambará, perifíton, rodovia AlexandraMatinhos (PR-508), Colônia Cambará, 1/IV/2000, $M$. Landucci \& E.L. Corrêa (UPCB44543); fitoplâncton, rodovia Alexandra-Matinhos (PR-508), Colônia Cambará, 1/IV/2000, M. Landucci \& E.L. Corrêa (UPCB44544); Guaratuba, rio Cabaraquara, perifíton, loc. de Cabaraquara, próximo à PR-412, 2/IV/2000, M. Landucci \& D. Atab (UPCB44547).

2. Aulacoseira ambigua (Grunow) Simonsen var. ambigua, Bacillaria 2: 56. 1979.

Fig. 19.

Frústulas cilíndricas em vista pleural, formando cadeias retas, unidas por coroa marginal de espinhos curtos; presença de sulco profundo em forma de "U" e pseudosulco distinto; manto ornamentado por estrias areoladas, dispostas obliquamente em relação ao eixo pervalvar. Eixo pervalvar: 19,4-35,6 um; diâmetro: 4-7,2 $\mu \mathrm{m}$; estrias: 16-28 em $10 \mu \mathrm{m}$; aréolas: 22-26 em $10 \mu \mathrm{m}$. 
A forma típica difere da forma spiralis, que se apresenta em cadeias helicoidais. Os espécimes de Aulacoseira ambigua (Grunow) Simonsen foram encontrados em amostras planctônicas e perifíticas.

Material examinado: BRASIL. Paraná: Morretes, rio Nhundiaquara, fitoplâncton, Vila Freitas, rodovia PR-408, 25/III/2000, M. Landucci \& E.L. Corrêa (UPCB44536); Antonina, rio do Nunes, fitoplâncton, rodovia PR-340, 25/III/2000, M. Landucci \& E.L. Corrêa (UPCB44538); Paranaguá, rio Cambará, perifíton, rodovia Alexandra-Matinhos (PR-508), Colônia Cambará, 1/IV/2000, M. Landucci \& E.L. Corrêa (UPCB44543); fitoplâncton, rodovia Alexandra-Matinhos (PR-508), Colônia Cambará, 1/IV/2000, M. Landucci \& E.L. Corrêa (UPCB44544); Guaratuba, rio Cabaraquara, perifíton, loc. de Cabaraquara, próximo à PR-412, 2/IV/2000, M. Landucci \& D. Atab (UPCB44547).

3. Aulacoseira distans (Ehrenberg) Simonsen var. distans, Bacillaria 2: 57. 1979.

Fig. 14-16.

Frústulas cilíndricas em vista pleural, formando cadeias retas, curtas, unidas por espinhos de ligação curtos, inconspícuos; presença de sulco e pseudosulco pouco profundos; manto ornamentado por estrias areoladas delicadas, dispostas paralelamente em relação ao eixo pervalvar. Em vista valvar as aréolas estão presentes em toda superfície da valva. Eixo pervalvar: 6,4-11,8 $\mu \mathrm{m}$; diâmetro: 4,8-5,6 $\mu \mathrm{m}$; estrias: 17-20 em $10 \mu \mathrm{m}$.

Primeira citação de ocorrência do táxon para o Estado do Paraná, tendo sido encontrado em amostras planctônicas e perifíticas. A identificação baseou-se em Krammer \& Lange-Bertalot (1991). Mais comentários, ver Aulacoseira alpigena.

Material examinado: BRASIL. Paraná: Paranaguá, rio Cambará, perifíton, rodovia AlexandraMatinhos (PR-508), Colônia Cambará, 1/IV/2000, $M$. Landucci \& E.L. Corrêa (UPCB44543); fitoplâncton, rodovia Alexandra-Matinhos (PR-508), Colônia Cambará, 1/IV/2000, M. Landucci \& E.L. Corrêa (UPCB44544); Guaratuba, rio Cabaraquara, perifíton, loc. de Cabaraquara, próximo à PR-412, 2/IV/2000, M. Landucci \& D. Atab (UPCB44547).

4. Aulacoseira granulata var. angustissima (O. Müller) Simonsen, Bacillaria 2: 58. 1979.

Fig. 23-24.

Frústulas cilíndricas, em vista pleural, formando cadeias retas, unidas por espinhos de ligação curtos e um único longo; presença de sulco e pseudosulco pouco evidentes; manto ornamentado por estrias areoladas, paralelas a levemente oblíquas em relação ao eixo pervalvar. Eixo pervalvar: 24,2-36,2 $\mu \mathrm{m}$; diâmetro: 2,4-3,2 $\mu \mathrm{m}$; estrias: 14-20 em $10 \mu \mathrm{m}$; aréolas; 20-22 em $10 \mu \mathrm{m}$.

Hustedt (1927-1930) e Hubber-Pestalozzi (1942) difereciam esta variedade da variedade típica da espécie pelo diâmetro valvar menor e pelas estrias do manto delicadamente areoladas. Os exemplares estudados foram encontrados em amostras planctônicas e perifíticas.

Material examinado: BRASIL. Paraná: Morretes, rio Nhundiaquara, fitoplâncton, Vila Freitas, rodovia PR-408, 25/III/2000, M. Landucci \& E.L. Corrêa (UPCB44536); Paranaguá, rio Cambará, perifíton, rodovia Alexandra-Matinhos (PR-508), Colônia Cambará, 1/IV/2000, M. Landucci \& E.L. Corrêa (UPCB44543); fitoplâncton, rodovia AlexandraMatinhos (PR-508), Colônia Cambará, 1/IV/2000, $M$. Landucci \& E.L. Corrêa (UPCB44544).

5. Aulacoseira granulata (Ehrenberg) Simonsen var. granulata, Bacillaria 2: 58. 1979.

Fig. 21-22.

Frústulas cilíndricas em vista pleural, formando cadeias retas, unidas por espinhos de ligação curtos e longos; presença de sulco e pseudosulco distintos, em forma de "V", manto ornamentado com estrias areoladas, grosseiras, paralelas a levemente oblíquas em relação ao eixo pervalvar. Eixo pervalvar:

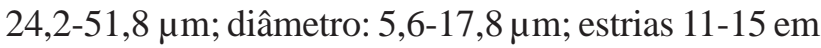
$10 \mu \mathrm{m}$; aréolas: 20-22 em $10 \mu \mathrm{m}$.

A presença de um longo espinho de ligação, juntamente com a presença de estrias grosseiras, são características importantes para a taxonomia deste grupo em nível específico. A espécie foi registrada em amostras planctônica e perifítica.

Material examinado: BRASIL. Paraná: Matinhos, rio da Onça, perifíton, reserva Parque Rio da Onça (nascente), 1/IV/2000, M. Landucci \& E.L. Corrêa (UPCB44545); Guaratuba, rio Cabaraquara, fitoplâncton, loc. de Cabaraquara, próximo à PR-412, 2/IV/2000, M. Landucci \& D. Atab (UPCB44548).

Classe Fragilariophyceae Round

Ordem Fragilariales Silva

Família Fragilariaceae Greville

Asterionella Hassal 

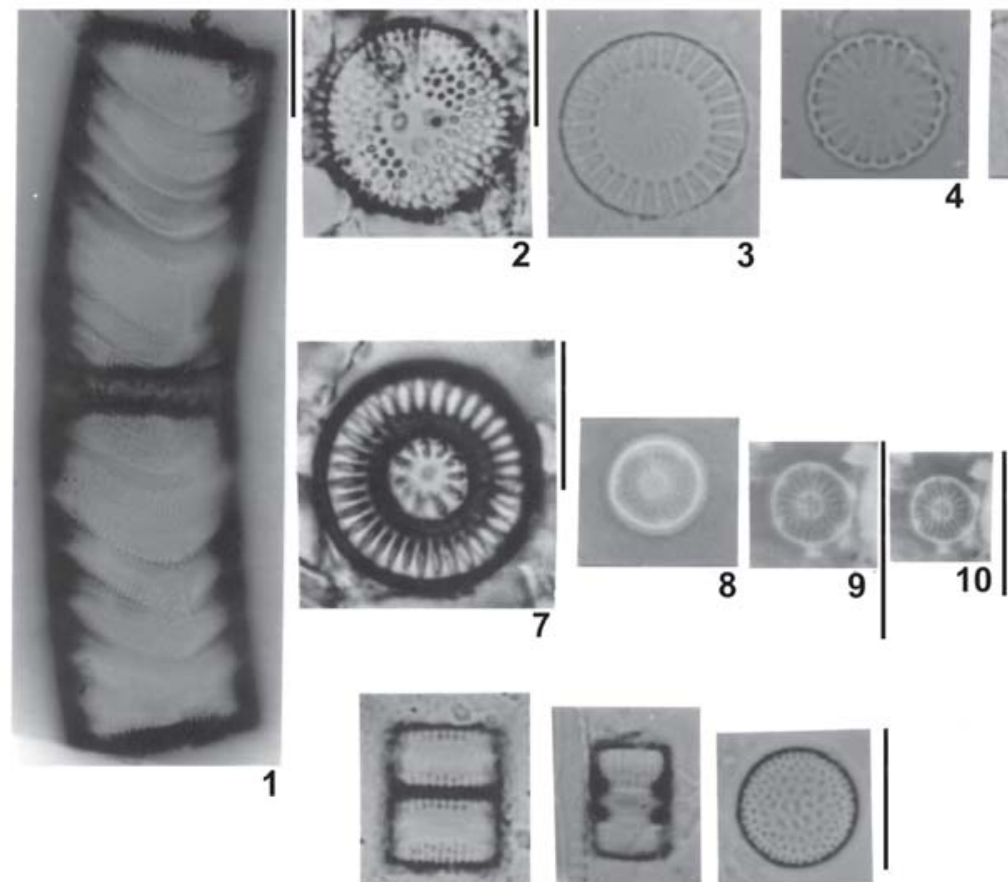

14
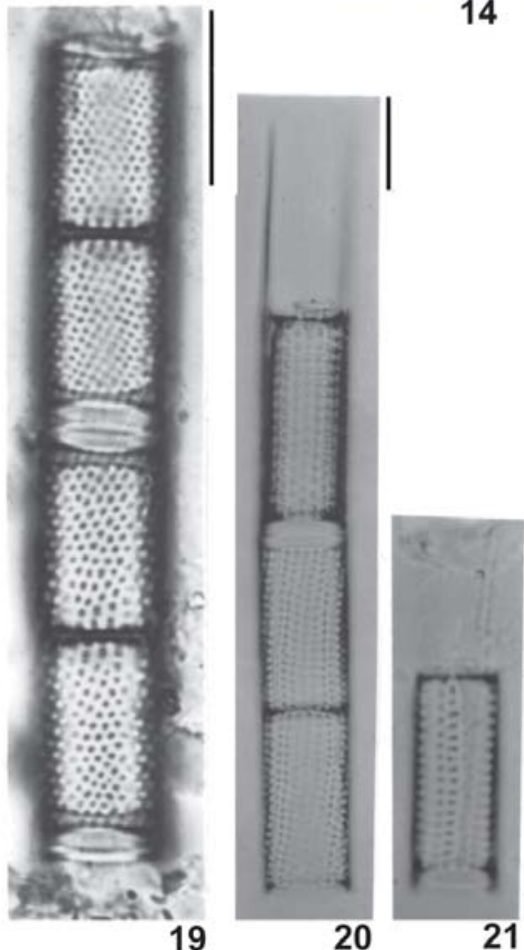

21
15

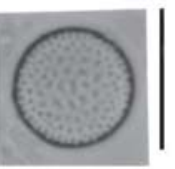

16
4
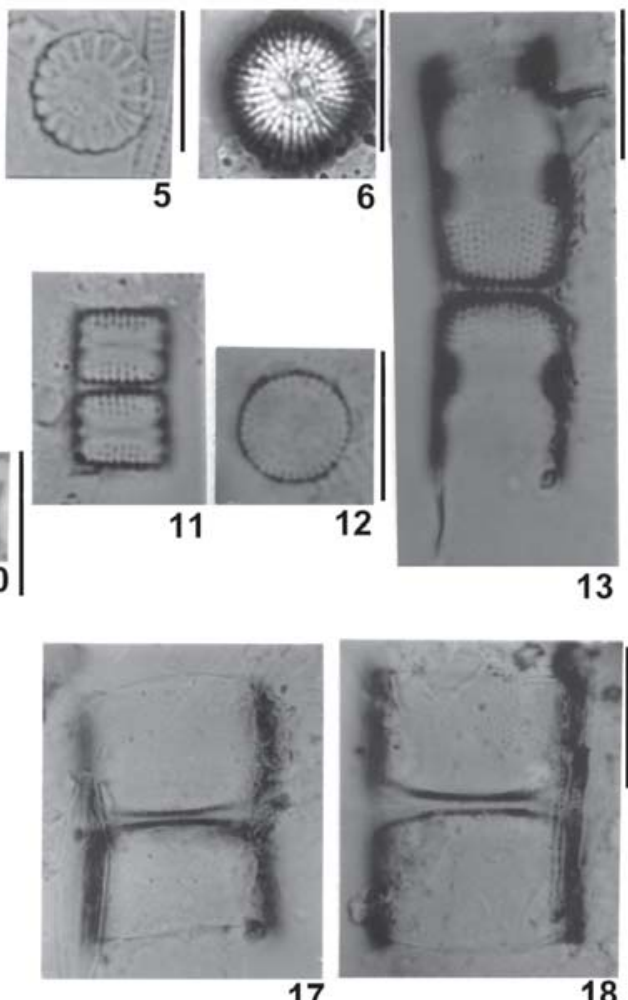

17

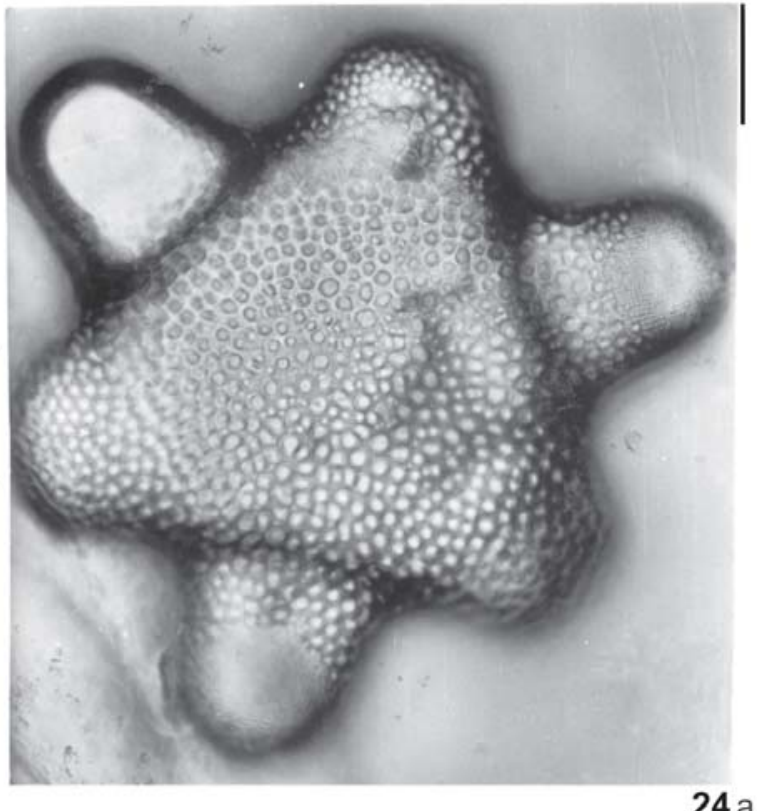

Figuras 1-24a. Diatomáceas cêntricas da bacia Litorânea paranaense, Paraná, Brasil. 1-2. Orthoseira roeseana O’Meara var. roeseana; 3-5. Cyclotella meneghiniana Kützing var. meneghiniana; 6 e 13. Orthoseira dendroteres (Ehrenberg) Crawford var. dendroteres; 7. Cyclotella stelligera (Cleve \& Grunow) Van Heurck var. stelligera; 8-10. Cyclotella pseudostelligera Hustedt. var. pseudostelligera; 11-12. Aulacoseira alpigena (Grunow) Krammer var. alpigena; 14-16. Aulacoseira distans (Ehrenberg) Simonsen var. distans; 17-18. Melosira varians Agardh var. varians; 19. Aulacoseira ambigua (Grunow) Simonsen var. ambigua f. ambigua; 20-21. Aulacoseira granulata Ehrenberg Simonsen var. granulata; 22-23. Aulacoseira granulata var. angustissima (O. Müller) Simonsen; 24a. Hydrosera whampöensis (Schwartz) Deby var. whampöensis. Escalas $=10 \mu \mathrm{m}$. 
Asterionella formosa Hassal var. formosa, Micr. Exam. Water, p. 10, pl. 2, fig. 5. 1855.

Fig. 25-26.

Valvas lineares, iso ou heteropolares, extremidades capitadas; área axial estreita, linear, inconspícua; área central ausente; estrias paralelas, pouco visíveis. Eixo apical: 48,6-86,6 $\mu \mathrm{m}$; eixo transapical: 2,4-3,2 $\mu \mathrm{m}$; estrias: $20-26$ em $10 \mu \mathrm{m}$.

Krammer \& Lange-Bertalot (1991) sinonimizaram em $A$. formosa Hassal as espécies $A$. formosa var. gracillima (Hantzsch) Grunow e A. gracillima (Hantzsch) Heiberg. Esta última caracteriza-se pela heteropolaridade menos perceptível. Deste modo, os exemplares da bacia Litorânea que apresentaram maior isopolaridade foram incluídas na espécie típica, ressaltando-se ser esta, a primeira vez em que formas isopolares em $A$. formosa são registradas para o Estado do Paraná. Os exemplares identificados concordam com os descritos por Krammer \& Lange-Bertalot (1991), Van Heurck (1980-1981). A. formosa Hassal ocorreu em amostra fitoplanctônica.

Material examinado: BRASIL. Paraná: Paranaguá, rio do Salto, fitoplâncton, loc. de Quintilha, Rodovia PR-508, 1/IV/2000, M. Landucci \& E.L. Corrêa (UPCB44542).

\section{Fragilaria Lyngbye}

\section{Chave para os táxons de Fragilaria}

1. Valvas não intumescidas na região mediana 5. F. javanica var. javanica

1. Valvas intumescidas na região mediana

2. Valvas intumescidas unilateralmente 4. F. capucina var. vaucheriae

2. Valvas intumescidas bilateralmente

3. Valvas fortemente silicificadas

2. F. capucina var. fragilarioides

3. Valvas pouco silicificadas

4. Extremidades da valva subcapitadas

4. Extremidades da valva capitadas

1. F. capucina var. capucina 3. F. capucina var. gracilis

1. Fragilaria capucina Desmazières var. capucina, Plant. Cryptog. de la France, Fasc. (10), n. 453. 1825.

Fig. 39-41.

Valvas linear a linear-lanceoladas, pouco silicificada; extremidades subcapitadas; área axial linear; área central retangular, fortemente intumescida bilateralmente; estrias paralelas, delicadamente areoladas, que se intercalam às margens da valva. Eixo apical: 32,4-55,8 $\mu \mathrm{m}$; eixo transapical: 2,8-5 $\mu \mathrm{m}$; estrias: 16-18 em $10 \mu \mathrm{m}$.

Ludwig \& Flôres (1997) mencionaram que LangeBertalot, em 1980, após analisar o material-tipo de Synedra rumpens Kützing e de Fragilaria capucina Desmazières, concluíu que se tratavam de formas homólogas, enquadrando-as na sua forma mais antiga, Fragilaria capucina Desmazières.

Ludwig \& Flôres (1997) tentaram elucidar a problemática taxonômica que envolve o grupo Fragilaria capucina, levando em conta características como contorno valvar, número de estrias em10 mm e variações da área central. Krammer \& Lange-Bertalot (1991) realizaram um rearranjo do complexo Synedra rumpens dentro da espécie Fragilaria capucina, apresentando os grupos e suas características específicas, bem com a justificativa de suas variedades. O complexo Fragilaria capucina é bastante polimórfico, ressaltando-se assim, a necessidade de consultar vários autores como: Van Heurck (18801881); Patrick \& Reimer (1966), Lange-Bertalot (1980); Williams \& Round (1987); Jensen (1985); Krammer \& Lange-Bertalot (1991) e Germain (1981), a fim de melhor enquadrar as quatro variedades na população encontrada. A espécie foi registrada em amostras planctônica e perifítica.

Material examinado: BRASIL. Paraná: Paranaguá, rio Cambará, fitoplâncton, rodovia AlexandraMatinhos (PR-508), Colônia Cambará, 1/IV/2000, $M$. Landucci \& E.L. Corrêa (UPCB44544); Guaratuba, rio Cabaraquara, perifíton, loc. de Cabaraquara, próximo à PR-412, 02/IV/2000, M. Landucci \& D. Atab (UPCB44547).

2. Fragilaria capucina var. fragilarioides (Grunow) Ludwig \& Flores, Hoehnea, 24(1): 55-65. 1997.

Fig. 27-31.

Valvas linear-lanceoladas, fortemente silicificadas; 
extremidades capitadas a subcapitadas; área axial linear, estreita; área central retangular, intumescida bilateralmente; estrias paralelas, grosseiramente areoladas, que se intercalam às margens da valva. Eixo apical: 35,2-66,4 $\mu \mathrm{m}$; eixo transapical: 3,2-6,4 $\mu \mathrm{m}$; estrias: 11-14 em $10 \mu \mathrm{m}$.

Com base no descrito por Patrick \& Reimer (1966) e Jensen (1985), para Synedra rumpens var. fragilarioides Grunow, Ludwig \& Flôres (1997), estabeleceram alguns critérios taxonômicos para proposição de uma nova combinação: Fragilaria capucina var. fragilarioides. Estes critérios estavam relacionados ao contorno valvar, podendo variar de linear a linear-lanceolado, presença de 9-14 estrias grosseiras e área central distinta, intumescida bilateralmente. O material estudado presente em amostras planctônicas e perifíticas concorda com o proposto por Ludwig \& Flôres (1997), apresentando pouca variação morfológica no padrão de estrias, mas destacada variação métrica do eixo apical.

Material examinado: BRASIL. Paraná: Morretes, rio Iporanga, fitoplâncton, loc. de América de Cima, próximo à estação de tratamento de água da SANEPAR 18/III/2000, M. Landucci \& E.L. Corrêa (UPCB44534); rio Nhundiaquara, perifíton, Vila Freitas, rodovia PR-408, 25/III/2000, M. Landucci \& E.L. Corrêa (UPCB44535); fitoplâncton, Vila Freitas, rodovia PR-408, 25/III/2000, M. Landucci \& E.L. Corrêa (UPCB44536); Antonina, rio do Nunes, fitoplâncton, rodovia PR-340, 25/III/2000, M. Landucci \& E.L. Corrêa (UPCB44538); perifíton, loc.de Bairro Alto, 25/III/2000, M. Landucci \& E.L. Corrêa (UPCB44539); idem, Paranaguá, rio do Salto, perifíton, loc.de Quintilha, Rodovia PR-508, 1/IV/2000, $M$. Landucci \& E.L. Corrêa (UPCB44541); fitoplâncton, loc. de Quintilha, Rodovia PR-508, 1/IV/2000, $M$. Landucci \& E.L. Corrêa (UPCB44542); Cambará, perifíton, rodovia Alexandra-Matinhos (PR-508), Colônia Cambará, 1/IV/2000, M. Landucci \& E.L. Corrêa (UPCB44543); fitoplâncton, rodovia AlexandraMatinhos (PR-508), Colônia Cambará, 1/IV/2000, $M$. Landucci \& E.L. Corrêa (UPCB44544).

3. Fragilaria capucina var. gracilis (Oestrup) Hustedt, Arch. für Hidrobiol., p. 43, pl. 36, fig. 31. 1950.

Fig. 24b.

Valvas lineares a lanceladas, pouco silicificadas; extremidades capitadas; área axial linear, estreita; área central retangular, geralmente, intumescida bilateralmente; estrias paralelas, delicadamente areoladas, que se intercalam às margens da valva. Eixo apical: 19,4-25,9 $\mu \mathrm{m}$; eixo transapical: 2,4-3,2 $\mu \mathrm{m}$; estrias: $16-18$ em $10 \mu \mathrm{m}$.

Ludwig \& Flôres (1997) incluiram os dois tipos morfológicos encontrados na população da usina hidrelétrica de Segredo no mesmo grupo, Fragilaria capucina Desmazières var. gracilis (Oestrup) Hustedt, por apresentarem grande semelhança no padrão delicado das estrias, pequeno intumescimento na região mediana e contorno valvar linear-lanceolado, considerando estas características taxonomicamente importantes para a espécie discutida. A espécie foi encontrada em amostras planctônicas e perifíticas, concordando com os apresentados por Patrick \& Reimer (1966), Krammer \& Lange-Bertalot (1991) e Ludwig \& Flôres (1997).

Material examinado: BRASIL. Paraná: Morretes, rio Nhundiaquara, perifíton, Vila Freitas, rodovia PR-408, 25/III/2000, M. Landucci \& E.L. Corrêa (UPCB44535); fitoplâncton, Vila Freitas, rodovia PR408, 25/III/2000, M. Landucci \& E.L. Corrêa (UPCB44536); Antonina, rio Cachoeira, fitoplâncton, loc.de Bairro Alto, 25/III/2000, M. Landucci \& E.L. Corrêa (UPCB44540); Paranaguá, rio do Salto, perifíton, loc.de Quintilha, Rodovia PR-508, 1/IV/2000, M. Landucci \& E.L. Corrêa (UPCB44541); fitoplâncton, loc. de Quintilha, Rodovia PR-508, 1/IV/2000, M. Landucci \& E.L. Corrêa (UPCB44542); Cambará, perifíton, rodovia AlexandraMatinhos (PR-508), Colônia Cambará, 1/IV/2000, M. Landucci \& E.L. Corrêa (UPCB44543).

4. Fragilaria capucina var. vaucheriae (Kützing) Lange-Bertalot, Hedwigia, 33: 747, pl. 1, fig. 26-38; pl. 4, fig. 82-94, 97-102; pl. 11, fig. 216, 224; pl. 12, fig. 225, 233. 1980.

Fig. 32-33.

Valvas lanceoladas a linear lanceoladas; extremidades subcapitadas a rostradas; área axial levemente lanceolada a linear; área central, intumescida unilateralmente; estrias paralelas, que se intercalam às margens da valva. Eixo apical: 10,5-25,9 $\mu \mathrm{m}$; eixo transapical: 3,2-6,4 $\mu \mathrm{m}$; estrias: 10-15 em $10 \mu \mathrm{m}$.

Os exemplares encontrados apenas em amostras perifíticas apresentaram pouco polimorfismo com relação às extremidades e ao contorno valvar, mantendo-se constante a assimetria na área central, devido à presença de um intumescimento unilateral. Este intumescimento, segundo Patrick \& Reimer (1966) é uma característica taxonômica importante para $F$. vaucheriae (Kützing) Petersen, assim como para Jensen (1985), Krammer \& Lange-Bertalot (1991) e 
Ludwig \& Flôres (1997). O material examinado concorda com as características morfométricas do material apresentado por Patrick \& Reimer (1966); Jensen (1985) e Krammer \& Lange-Bertalot (1991).

Material examinado: BRASIL. Paraná: Morretes, rio Iporanga, perifíton, loc. de América de Cima, próximo à estação de tratamento de água da SANEPAR, 18/III/2000, M. Landucci \& E.L. Corrêa (UPCB44533); Antonina, rio do Nunes, perifíton, rodovia PR-340, 25/III/2000, M. Landucci \& E.L. Corrêa (UPCB44537); rio Cachoeira, perifíton, loc.de Bairro Alto, 25/III/2000, M. Landucci \& E.L. Corrêa (UPCB44539).

5. Fragilaria javanica Hustedt var. javanica, Arch. Hydrobiol., 6(1): pl. 1, fig. 59-60. 1938.

Fig. 36-38.

Valvas lineares, linear-lanceoladas a lanceoladas, podendo ou não apresentar constrição na região mediana; extremidades capitadas a subcapitadas; áreas axial e central ausentes; estrias delicadamente areoladas, paralelas em toda extensão da valva. Eixo apical: 22,6 a 69,6 $\mu \mathrm{m}$; eixo transapical: 3,6 a 7,2 $\mu \mathrm{m}$; estrias 16 a 28 em $10 \mu \mathrm{m}$.

O material analisado, encontrado em amostras planctônicas e perifíticas, apresentou uma ampla variação morfológica em relação ao contorno valvar, desde linear, linear-lanceolado a lanceolado, com e sem constrição mediana; e estrias delicada a grosseiramente areoladas. Esta espécie apresenta características que justificam sua transferência para o gênero Fragilariforma. Entretanto, Williams \& Round (1987), ao transferirem Synedra strangulata Zanon, 1938, para o gênero citado, comentaram a estreita relação entre esta espécie e Fragilaria javanica Hustedt, 1938. Os autores salientaram a necessidade de estudos taxonômicos adicionais para esclarecer a identidade das espécies, com verificação de materiais-tipo. Os exemplares registrados concordam com os apresentados por Krammer \& Lange-Bertalot (1991) e Metzeltin \& Lange-Bertalot (1998).

Material examinado: BRASIL. Paraná: Morretes, rio Iporanga, perifíton, loc. de América de Cima, próximo à estação de tratamento de água da SANEPAR, 18/III/2000, M. Landucci \& E.L. Corrêa (UPCB44533); fitoplâncton, loc. de América de Cima, próximo à estação de tratamento de água da SANEPAR 18/III/2000, M. Landucci \& E.L. Corrêa (UPCB44534); rio Nhundiaquara, perifíton, Vila Freitas, rodovia PR-408, 25/III/2000, M. Landucci \& E.L. Corrêa (UPCB44535); fitoplâncton, Vila Freitas, rodovia PR-408, 25/III/2000, M. Landucci \& E.L. Corrêa (UPCB44536); Antonina, rio Cachoeira, fitoplâncton, loc.de Bairro Alto, 25/III/2000, M. Landucci \& E.L. Corrêa (UPCB44540); Paranaguá, rio do Salto, fitoplâncton, loc. de Quintilha, Rodovia PR-508, 1/IV/2000, M. Landucci \& E.L. Corrêa (UPCB44542); rio Cambará, fitoplâncton, rodovia Alexandra-Matinhos (PR-508), Colônia Cambará, 1/IV/2000, M. Landucci \& E.L. Corrêa (UPCB44544); Matinhos, rio da Onça, perifíton, reserva Parque Rio da Onça (nascente), 1/IV/2000, M. Landucci \& E.L. Corrêa (UPCB44545); fitoplâncton, reserva Parque Rio da Onça (nascente), 1/IV/2000, M. Landucci \& E.L. Corrêa (UPCB44546); Guaratuba, rio Cabaraquara, perifíton, loc. de Cabaraquara, próximo à PR-412, 2/IV/2000, M. Landucci \& D. Atab (UPCB44547); fitoplâncton, loc. de Cabaraquara, próximo à PR-412, 2/IV/2000, M. Landucci \& D. Atab (UPCB44548).

\section{Staurosira Ehrenberg}

Staurosira construens Ehrenberg var. construens, Phys. Abh. Wiss. Berl., for: 1841: 424. 1843.

Fig. 34-35.

Valvas linear-lanceoladas, intumescidas bilateralmente; extremidades arredondadas; área axial estreita, linear; área central ausente; estrias transapicais indistintamente areoladas, radiadas em toda superfície valvar. Eixo apical: 4,8-11,9 $\mu \mathrm{m}$; eixo transapical: 3,2-5,6 $\mu \mathrm{m}$; estrias 10-12 em $10 \mu \mathrm{m}$.

Segundo Patrick \& Reimer (1966), Krammer \& Lange-Bertalot (1991) e Germain (1981), Fragilaria construens, apresenta um acentuado polimorfismo com relação ao contorno valvar variando de linear, linear-lanceolado, rômbico-lanceolado a elíptico. No entanto, no material referente à bacia Litorânea, constatou-se apenas tipos morfológicos com contorno valvar linear-lanceolado, apresentando intumescimento bilateral. Os exemplares estudados foram encontrados em amostras planctônicas e perifíticas e se enquadram na circunscrição de Staurosira construens (Ehrenberg) Williams \& Round estudados por Patrick \& Reimer (1966), Krammer \& Lange-Bertalot (1991) e Germain (1981).

Material examinado: BRASIL. Paraná: Antonina, rio Cachoeira, perifíton, loc.de Bairro Alto, 25/III/2000, M. Landucci \& E.L. Corrêa (UPCB44539); Matinhos, rio da Onça, perifíton, reserva Parque Rio da Onça (nascente), 1/IV/2000, M. Landucci \& E.L. Corrêa (UPCB44545); fitoplâncton, reserva Parque Rio da 

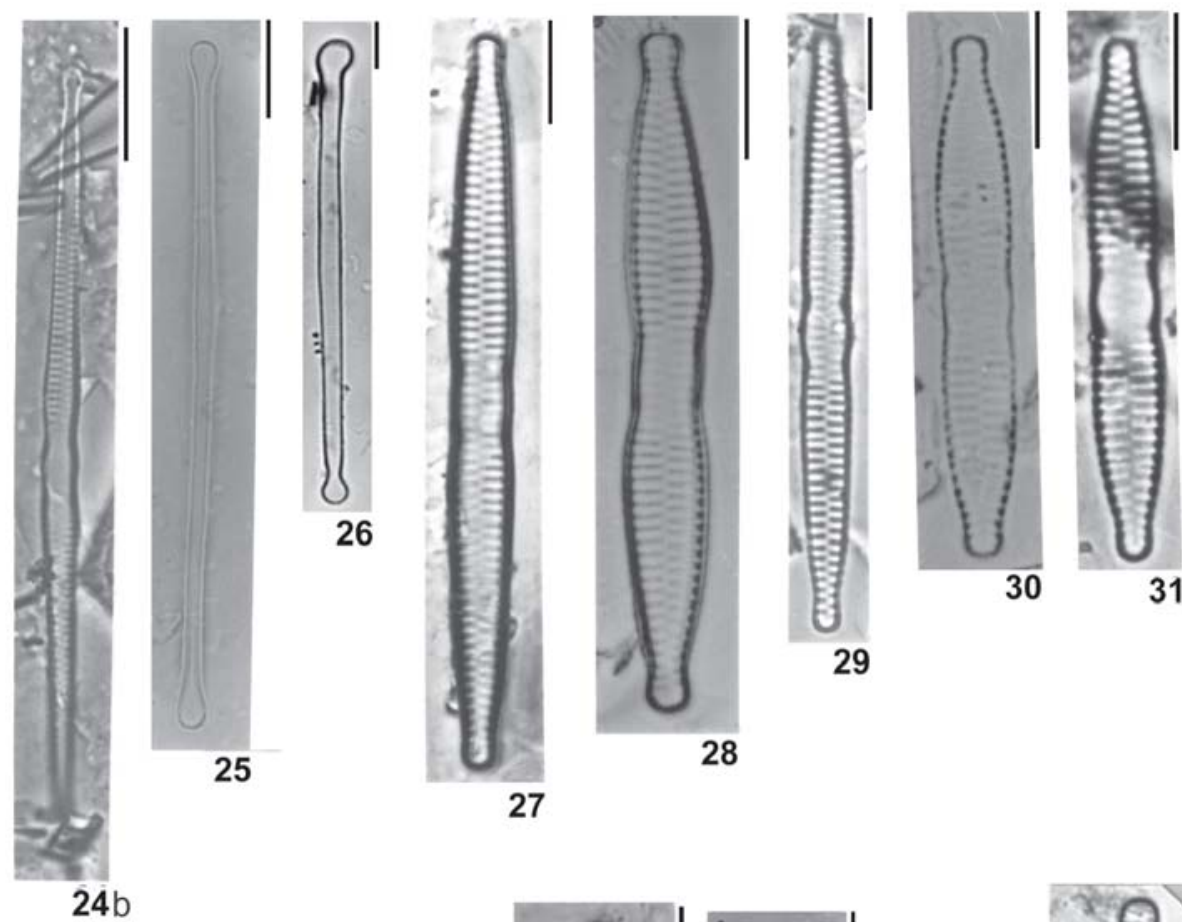

29

$$
27
$$
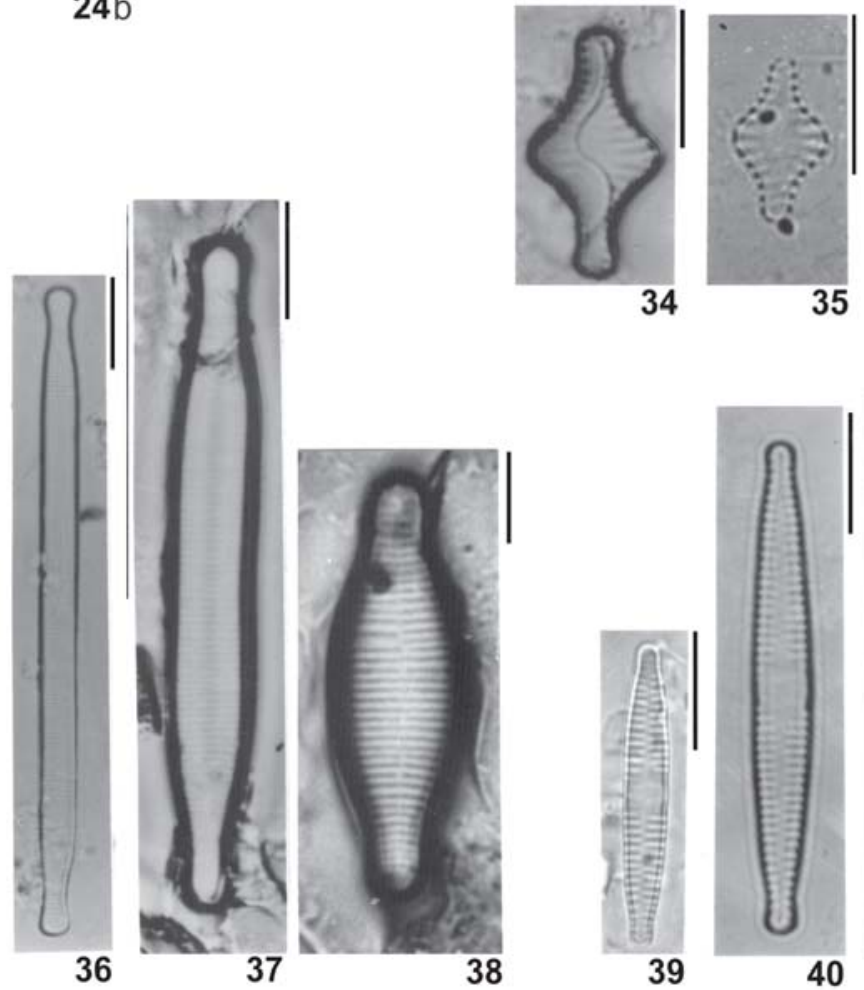

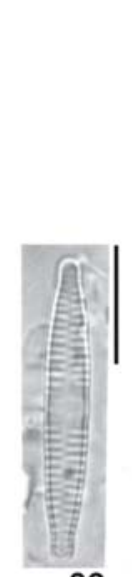

39

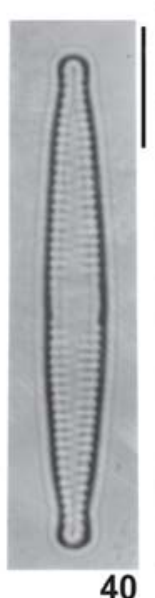

40
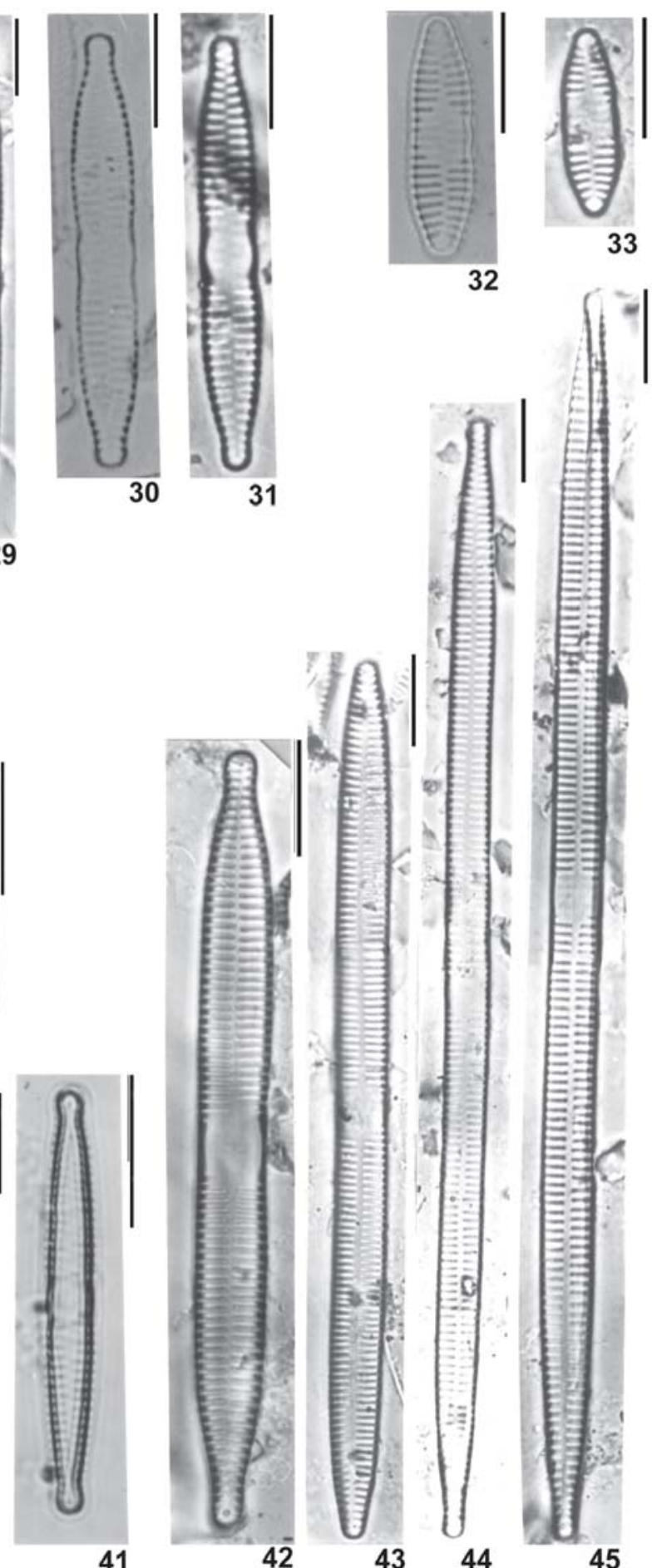

33

32
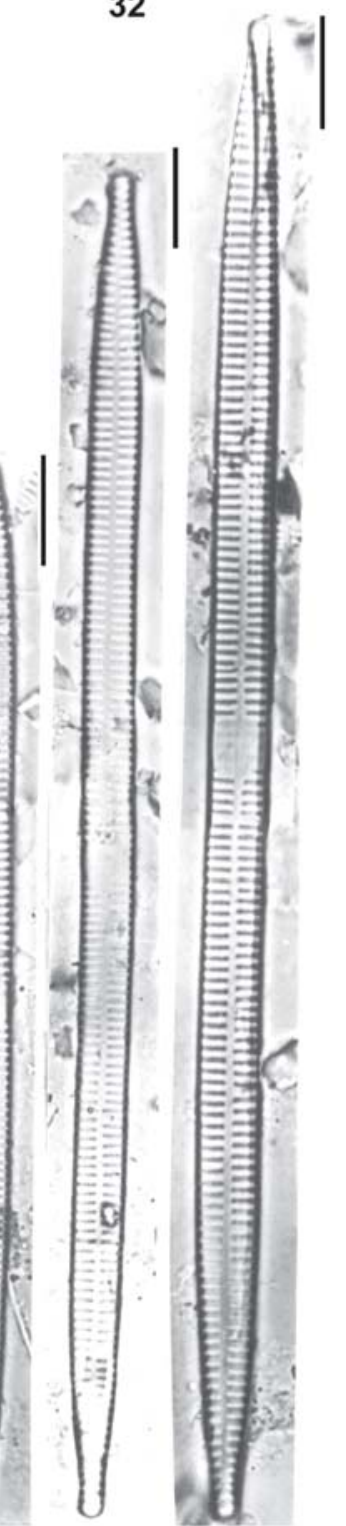

34

45

Figuras 24b-45. Diatomáceas da bacia Litorânea paranaense, Paraná, Brasil. 24b. Fragilaria capucina (Oestrup) Husredt var. gracilis. 25-26. Asterionella formosa Hassal var. formosa. 27-31. Fragilaria capucina Desmazières var. fragilarioides. 32-33. Fragilaria capucina var. vaucheriae (Kützing) Lange-Bertalot. 34-35. Staurosira construens Ehrenberg var. construens. 36-38. Fragilaria javanica Hustedt var. javanica. 39-41. Fragilaria capucina Desmazières var. capucina. 42-45. Ulnaria ulna (Nitzsch) Compère var. ulna. Escalas = 10 um. 
Onça (nascente), 1/IV/2000, M. Landucci \& E.L. Corrêa (UPCB44546).

\section{Ulnaria (Kützing) Compère}

Ulnaria ulna (Nitzsch) Compère var. ulna, in Jahn et al., Studies on diatoms, 97-101. 2001.

Fig. 42-45.

Valvas lineares a linear-lanceoladas; sem constrição mediana; extremidades rostradas a subcapitadas; área axial linear, área central retangular; estrias paralelas, justapostas a outra margem. Eixo apical: 73,7-163,6 $\mu \mathrm{m}$; eixo transapical: 4,8-6,6 $\mu \mathrm{m}$; estrias: $10-14$ em $10 \mu \mathrm{m}$.

Patrick \& Reimer (1966) apresentaram nove variedades, incluindo a variedade típica, diferenciando-as principalmente, pela forma das extremidades, contorno valvar, presença ou não de área central e a maneira como esta área central se dispõe. Porém, o acentuado polimorfismo relacionado ao contorno valvar e à forma da área central, não justifica as inúmeras variedades já propostas. A espécie foi observada em amostras planctônicas e perifíticas.

Material examinado: BRASIL. Paraná: Morretes, rio Nhundiaquara, perifíton, Vila Freitas, rodovia PR-408, 25/III/2000, M. Landucci \& E.L. Corrêa (UPCB44535); fitoplâncton, Vila Freitas, rodovia PR-408, 25/III/2000, M. Landucci \& E.L. Corrêa (UPCB44536); Antonina, rio do Nunes, perifíton, rodovia PR-340, 25/III/2000, M. Landucci \& E.L. Corrêa (UPCB44537); fitoplâncton, rodovia PR-340, 25/III/2000, M. Landucci \& E.L. Corrêa (UPCB44538); rio Cachoeira, perifíton, loc.de Bairro Alto, 25/III/2000, M. Landucci \& E.L. Corrêa (UPCB44539); Paranaguá, rio do Salto, perifíton, loc.de Quintilha, Rodovia PR-508, 1/IV/2000, M. Landucci \& E.L. Corrêa (UPCB44541); fitoplâncton, loc. de Quintilha, Rodovia PR-508, 1/IV/2000, M. Landucci \& E.L. Corrêa (UPCB44542); rio Cambará, perifíton, rodovia Alexandra-Matinhos (PR-508), Colônia Cambará, 1/IV/2000, M. Landucci \& E.L. Corrêa (UPCB44543); fitoplâncton, rodovia AlexandraMatinhos (PR-508), Colônia Cambará, 1/IV/2000, M. Landucci \& E.L. Corrêa (UPCB44544); Matinhos, Rio da Onça, fitoplâncton, reserva Parque Rio da Onça (nascente), 1/IV/2000, M. Landucci \& E.L. Corrêa (UPCB44546); Guaratuba, rio Cabaraquara, fitoplâncton, loc. de Cabaraquara, próximo à PR-412, 2/IV/2000, M. Landucci \& D. Atab (UPCB44548).

\section{Agradecimentos}

Os autores agradecem à Coordenadoria de Aperfeiçoamento de Pessoal de Nível Superior CAPES, pela concessão da bolsa de Mestrado à primeira Autora.

\section{Referências bibliográficas}

Bigarella, J.J. 1978. A Serra do Mar e a porção oriental do Estado do Paraná. Curitiba, Governo do Paraná Secretaria de Estado do Planejamento/ADEA.

Bittencourt-Oliveira, M.C. 2002. A comunidade fitoplanctônica do rio Tibagi: uma abordagem preliminar de sua diversidade. Pp. 373-402. In: M.E. Medri (ed.). A bacia do rio Tibagi. Londrina, M.E. Medri.

Brassac, N.M.; Atab, D.R.; Landucci, M.; Visinoni, N.D. \& Ludwig, T.V. 1999. Diatomáceas cêntricas de rios na região de abrangência da usina hidrelétrica de Salto Caxias, Pr (Bacia do Iguaçu). Acta Botanica Brasilica 13(3): 277-289.

Cleve-Euler, A. 1951. Die diatomeen von Scheweden und Finnland. Kunglika Svenska Vetenskapsakademiens Handlingar 2(1): 1-163.

Contin, L.F. 1990. Contribuição ao estudo das diatomáceas (Chrysophyta, Bacillariophyceae) na região de captação d’água do rio Iguaçu (SANEPAR), em Curitiba, Estado do Paraná, Brasil. Estudos de Biologia (24): 5-95.

Cox, E.J. 1996. Identification of the freshwater diatoms from live material. London, Chapmann \& Hall.

Deby, J. 1891. Note sur le genre Hydrosera de Wallich. Journal Micrographie 15: 209-212.

Domitrovic, Y. \& Maidana, N.I. 1997. Taxonomic and ecological studies of the Paraná River diatom flora (Argentina). Bibliotheca Diatomologica 34: 1-122.

Frenguelli, J. 1953. Diatomeas del territorio nacional de Missiones. Revista del Museu de La Ciudad Eva Péron, Botanica 8: 63-86.

Germain, H. 1981. Flore des diatomées. Paris, Boubée.

Houk, V. 1993. Some morphotypes in the "Orthoseira roeseana” complex. Diatom Research 8(2): 385-402.

Huber-Pestalozzi, G. 1942. Das Phytoplankton dês Süsswassers. Pp. 1-549. In: A. Thienemann (ed.). Die Binnengewässer. Stuttgart, E. Schweizerbart'sche Verlagsbunchhandlung.

Hustedt, F. 1927-1930. Die kieselalgen. In: Rabenhorst, L. Kryptogamen-Flora. Leipzig, Akademische Verlagsgesellschaft. v.5, n.2.

Jensen, N. C. 1985. Hustedt's “Die Kieselalgen, 2. Teil”: The Pennate Diatoms. Koenigstein, Koeltz Scientific Books.

Krammer, K. \& Lange-Bertalot, H. 1991. Bacillariophyceae: Achnanthaceae. In: H. Ettl; I. Gerloff; H. Heynig \& D. Mollenhauer (eds.). Süsswasser Flora von Mittleleuropa. Sttugart, G. Fischer. v.2, n.4.

Lange-Bertalot, H. 1980. Zur systematischen Bewertung der bandförmigen Kolonien bei Navicula und Fragilaria. Nova Hedwigia 33: 723-787. 
Leandrini, J.A.; Moreira Filho, H. \& Rodrigues, L. 2002. Espécies perifíticas de Navicula Bory de dois sistemas lóticos do município de Maringá, Estado do Paraná, Brasil. Hoehnea 29(1): 49-56.

Ludwig, T.A.V. \& Flôres, T. 1995. Diatomoflórula dos rios da região a ser inundada para a construção da Usina Hidrelétrica de Segredo, Paraná; I. Coscinodiscaceae, Bacillariophyceae (Achnanthales e Eunotiales) e Fragilariophyceae (Meridion e Asterionella). Arquivos de Biologia e Tecnologia 38(2): 631-650.

Ludwig, T.A.V. \& Flôres, T. 1997. Diatomoflórula dos rios da região a ser inundada para a construção da Usina Hidrelétrica de Segredo, Paraná Fragilariophyceae (Fragilaria e Synedra). Hoehnea 24(1): 55-65.

Metzeltin, D. \& Lange-Bertalot, H. 1998. Tropical Diatoms of the South America I. Iconographia Diatomologica 5: 1-695.

Moreira Filho, H. \& Valente-Moreira, I.M. 1981. Avaliação taxonômica e ecológica das diatomáceas (Bacillariophyceae) epífitas em algas pluricelulares obtidas nos litorais do estado do Paraná, Santa Catarina e São Paulo. Boletim do Museu Botânico Municipal (47): 1-17.
Patrick, R. \& Reimer, C.W. 1966. The diatoms of United States. Monographs of the Academy of Natural Sciences of Philadelphia 1: 1-688.

Round, F.E.; Crawford, R.M. \& Mann, D.G. 1990. The Diatoms: biology and morphology of the genera. Cambridge, Cambridge University Press.

Schmidt, A. 1913. Atlas der Diatomaceen-Kunde. Leipzig, Reisland, O.R. 1874-1959.

Simonsen, R. 1974. The diatom plankton of the Indian Ocean Expedition of R/V “Meteor”, 1964-65 Meteor Forschungsergebnisse Reihe D-Biologie 19: 1-66.

Spauling, S.A. \& Kociolek, J.P. 1998. The genus Orthoseira: ultrastructure and morphological variation in two species from Madagascar with comments on nomenclature in the genus. Diatom Research 13(1): 133-147.

Van Heurck, H. 1880-1885. Synopsis des diatomées de Belgique. Anver, L’Auteur.

Williams, D.M. \& Round, F.E. 1987. Revision of the genus Fragilaria. Diatom Research 2(2): 267-288. 